\title{
Hybrid nanoparticle and thin film SPR biosensor with a high figure of merit
}

\author{
Arash Farhang, Banafsheh Abasahl, Olivier J.F. Martin \\ Nanophotonics and Metrology Laboratory, Swiss Federal Institute of Technology Lausanne (EPFL), \\ 1015 Lausanne, Switzerland;
}

\begin{abstract}
Due to their extreme sensitivity to refractive index changes, surface plasmon resonance (SPR) sensors have long been established as extremely valuable tools for biosensing. In the past few years researchers have begun investigating various other metallic nanostructures as candidates for localized SPR (LSPR) sensing. Although LSPR is not nearly as sensitive to bulk refractive index changes as standard SPR, is has the advantage of being extremely sensitive to local refractive index changes, thereby providing detection on the level of a single molecule. In practice such sensitivity criterion is of paramount importance since the analyte layer under investigation is often only a few nanometers thick and deposited directly on the surface of the metal. Most desirable, however, is a sensor that retains the total integrated sensitivity of a traditional SPR sensor and at the same time localizes this sensitivity right at the sensor surface. For this reason, we have investigated a hybrid structure composed of a 2D Au nanoparticle array coupled to a $\mathrm{Au}$ film. We show that this structure, when excited in the Kretschmann configuration, retains to a surprising degree the total integrated sensitivity of an ideal SPR sensor and is able to concentrate that sensitivity within a few nanometers of the sensor surface, thereby yielding a hybrid sensor with the advantages of both LSPR and SPR sensing, i.e. both a high local sensitivity and a high figure of merit (FOM).
\end{abstract}

Keywords: LSPR, SPR, biosensor, hybrid sensor, figure of merit, nanoparticle, surface plasmon, plasmonic.

\section{INTRODUCTION}

In recent years, an explosion of research has been seen in the field of plasmonics. The properties of many nanoscale metallic structures have, for example, been extensively explored for use as integrated optical circuits ${ }^{1-15}$, solar energy harvesters ${ }^{16-21}$, and as a cure for cancer ${ }^{22-25}$. The backbone to the use of metallic nanostructures for such applications is the resonant excitation of surface plasmon polariton (SPP) waves at a metal dielectric interface. Over a decade ago already, the excitation of freely propagating SPP waves via total internal reflection at the top surface of a thin metal film on a glass substrate proved as a very effective means to optically detect minute concentrations of biological analyte ${ }^{26}$. This is due to the extreme shifts in the excitation wavelength of the SPP wave induced by changes in the refractive index on the top surface of the metal film. In addition to its simplicity, this configuration boasts an extremely high figure of merit (FOM) and is sensitive to refractive index changes hundreds of nanometers above the metal film. Consequently, this device is not so sensitive to local refractive index changes directly on top of the metal film surface. To this end, great research efforts have been made in designing surface plasmon biosensors, specifically localized surface plasmon resonance (LSPR) biosensors ${ }^{27-33}$ that detect refractive index changes with an extremely high sensitivity directly at the metal surface due to highly localized optical fields in the direct vicinity of the metallic structure. Still, in terms of a FOM defined via bulk refractive index change, ie. the total sensitivity integrated from the sensor surface into the bulk of the medium, no LSPR sensor compares to a conventional SPR sensor ${ }^{34}$, 35. In this work we combine LSPR sensor components together with SPR sensor components, thereby obtaining a hybrid SPR sensor that preserves, as much as is possible, the total integrated sensitivity of a conventional SPR sensor, but with the added high surface sensitivity of an LSPR sensor.

The outline of this paper is as follows. In sections 2 we present the sensor geometry and simulation method; in section 3 results of numerical simulations; in section 4 the fabrication process, and finally we conclude in section 6 .

Plasmonics in Biology and Medicine IX, edited by Tuan Vo-Dinh, Joseph R. Lakowicz, Proc. of SPIE Vol. 8234, 82340P · (C) 2012 SPIE · CCC code: 1605-7422/12/\$18 · doi: 10.1117/12.908941 


\section{SENSOR GEOMETRY AND SIMULATION METHOD}

The proposed geometry consists of a $200 \times 200 \mathrm{~nm}$ period array of Au nanodots spaced approximately s=20nm from the surface of a $\mathrm{t}=50 \mathrm{~nm}$ thick $\mathrm{Au}$ film on top of a $\mathrm{SiO}_{2}\left(\mathrm{n}_{\mathrm{SiO}_{2}}=1.46\right)$ substrate. A single unit cell of the structure is shown in Fig. 1. The nanodots are modeled as cylinders with a diameter $\mathrm{d}=50 \mathrm{~nm}$ and a height $\mathrm{h}=40 \mathrm{~nm}$. The spacing between the nanodots and $\mathrm{Au}$ film is a $\mathrm{SiO}_{2}$ cylinder of the same diameter as that of the nanodots. The dielectric permittivity of $\mathrm{Au}$ is taken from the data of Johnson and Christy ${ }^{36}$. The sensor is immerged in a water background $\left(\mathrm{n}_{\mathrm{b}}=1.333\right)$ and is excited via total internal reflection of a TM polarized planewave in the Kretschmann configuration. We chose this form of excitation as we wish to excite SPP waves in the same manner as a conventional SPR sensor in order to maintain a high total integrated sensitivity. The addition of Au nanodots directly above the Au surface provides a means to confine the evanescent SPP field directly on the sensor surface, particularly right in the vicinity of the nanodots ${ }^{37-40}$, rather than having it extend hundreds of nanometers above the surface. Due to limitations in fabrication, as will be seen in section 4, the nanodots are additionally modeled as essentially half domes (Fig. 2).

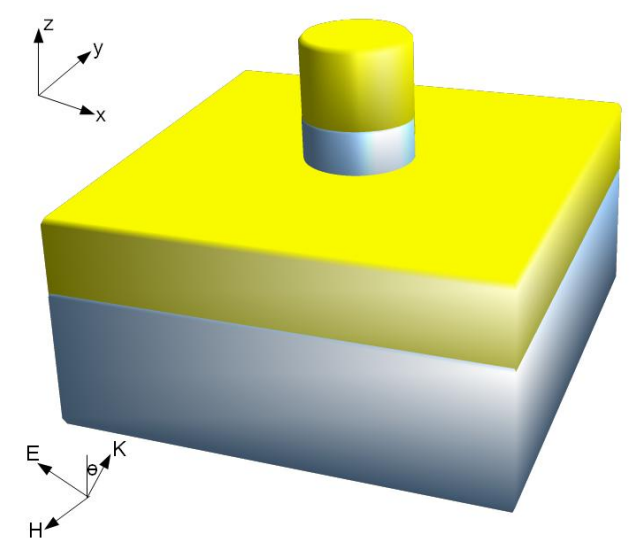

Figure 1. A single unit cell of the ideal sensor geometry, a cylindrical Au nanodot spaced above a thin Au film on a $\mathrm{SiO}_{2}$ substrate by an $\mathrm{SiO}_{2}$ spacer. The structure is excited via TM illumination. The periodicity is $200 \mathrm{x} 200 \mathrm{~nm}$ in $\mathrm{x}$ and $\mathrm{y}$ and the additional sensor parameters are given in the above text.

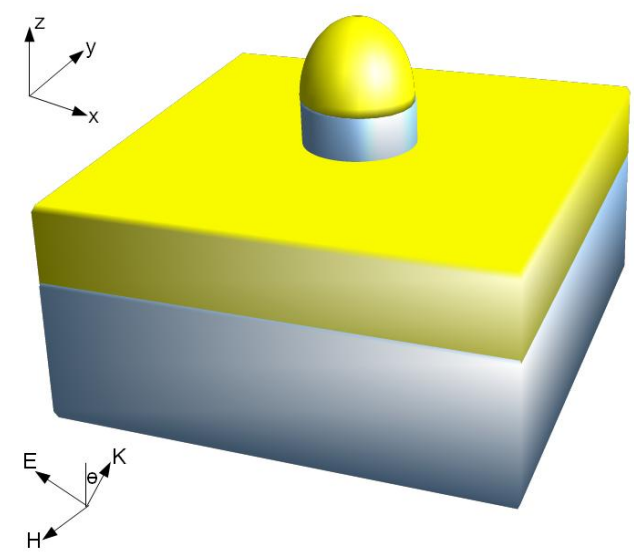

Figure 2. A single unit cell of the realistic sensor geometry, a half-dome Au nanodot spaced above a thin Au film. Apart from the shape, all parameters are the same as those of fig. 1.

The simulation method used in this study is based on the Surface Integral Equation (SIE) technique, extended for use in periodic structures ${ }^{41}$. It is particularly well suited as it allows for simulations of multilayer structures and only requires a meshing of the surfaces and an initial calculation of the surface currents, which can then be used to calculate the field arbitrarily close to or far away from the structure at a rapid pace. 


\section{NUMERICAL RESULTS}

\subsection{Ideal Geometry}

The chosen sensor geometry of Au nanodots above a Au film provides for high field confinement in the gap between the nanodots and film, particularly for very small nanodot film spacings of $\mathrm{s}<10 \mathrm{~nm}$. This is not ideal as it is this region that is filled with a $\mathrm{SiO}_{2}$ spacer and is thus inaccessible for sensing. Additionally such small spacings, provide very high levels of coupling between the nanodots and film, resulting in an anticrossing of the SPP dispersion and large redshifts in the resonances of interest ${ }^{37-39,42-45}$. These redshifts become even greater when the background is realistically modeled as water rather than air. What is needed is an optimal nanodot-film spacing that provides for a high field confinement on the nanodot surface rather than solely in the gap and which doesn't result in such significant redshifts of the resonance wavelength. It was found through numerical simulations that a spacing $\mathrm{s}=20 \mathrm{~nm}$ provides for an optimal level of field confinement on the nanodot surface, without compensating too much the high levels of localized field enhancement. We show in Fig. 3 the calculated reflection spectra of such a structure for angles of incidence $65-85^{\circ}$. As can be seen the reduced level of coupling does not produce an anticrossing, rather, the SPP dispersion curve is just redshifted from the case of a plain Au film (Fig. 4). Additionally, the electric field profile shown in Fig. 5 for a cross section in the x-z plane cutting directly through the center of the nanodot shows not only high field enhancement in the gap between the nanodot and film, but also on the nanodot surface, particularly at the corners.

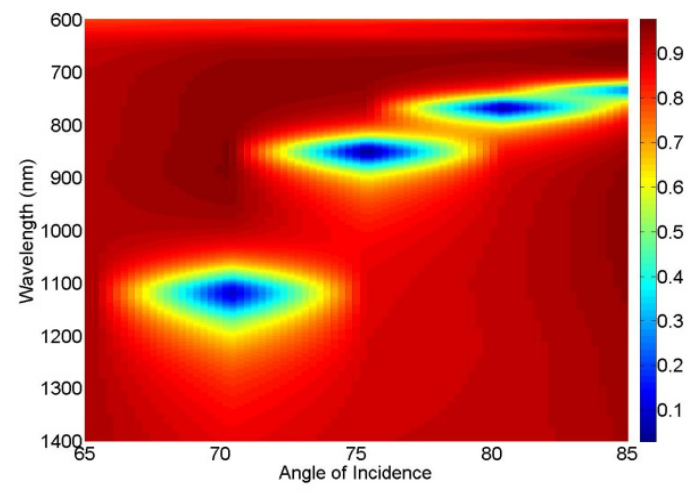

Figure 3. Reflection spectra of the structure shown in fig. 1 depicting the SPP dispersion. Note, the discrete jumps in the reflection spectra are solely due to the fact that simulations are only performed at $5^{\circ}$ increments due to the constraint in simulation time. The data is smooth nonetheless as a result of interpolation.

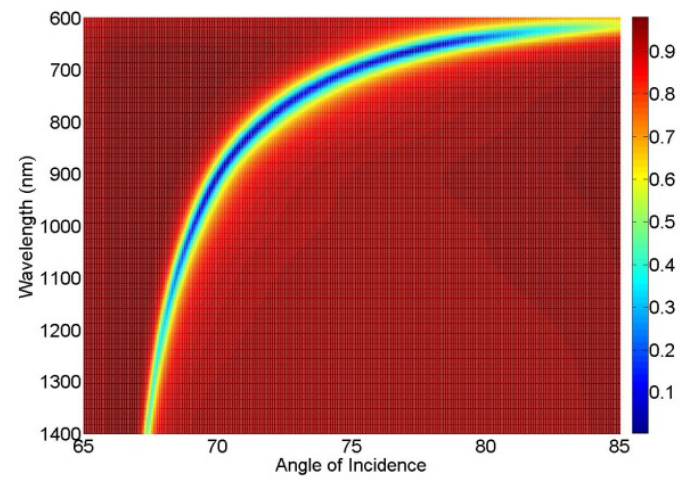

Figure 4. Reflection spectra for just a $50 \mathrm{~nm}$ thick $\mathrm{Au}$ film on a $\mathrm{SiO}_{2}$ substrate. In comparison with fig. 3 it can be seen that the Au nanodots of the hybrid sensor induce a redshift in the SPP dispersion of the Au film. The reflection spetra here is calculated via an analytical form of the transfer matrix method. 


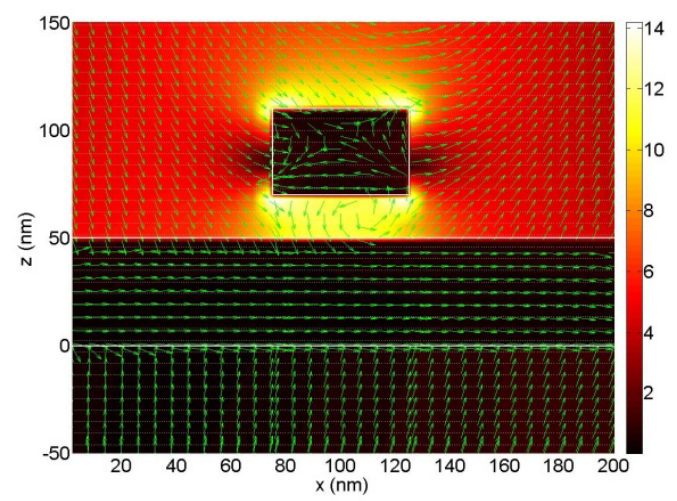

Figure 5. Electric field profile at $70^{\circ}$ incidence for a cross section in the $\mathrm{x}-\mathrm{z}$ plane cutting through the center of the cylindrical nanodot. Arrows depict the electric field orientation and the color scale shows the field magnitude.

As a benchmark we compare the sensitivity of this sensor to that of a traditional SPR sensor, ie. a plain Au film of $\mathrm{t}=50 \mathrm{~nm}$ thickness on top of an $\mathrm{SiO}_{2}$ substrate, all submerged in a water background. Note, although high bulk sensitivity is not the aim of this hybrid sensor, it is still a good measure in determining how well the total integrated sensitivity is conserved in comparison to a conventional SPR sensor. To this end we compare the shift of the SPP resonant dip for the case of a conventional SPR sensor and our hybrid sensor when the background index is altered slightly from $\mathrm{n}_{\mathrm{b}}=1.333$ to $\mathrm{n}_{\mathrm{b}}=1.34$ with a fixed angle of incidence. Note, unless otherwise noted, all numerical simulations for the hybrid sensor are for incidence angles of $70^{\circ}$ and $75^{\circ}$. Additionally, the base point of comparison for a plain Au film is taken such that the angles of incidence correspond to the same resonance wavelengths as that of the hybrid structure for a water background with $\mathrm{n}_{\mathrm{b}}=1.333$. A comparison of the results, summarized in table 1 , shows that the average wavelength shift $=\Delta \lambda, \Delta \lambda / \Delta \mathrm{n}$, and $\mathrm{FOM}=\Delta \lambda / \Delta \mathrm{n} * \mathrm{FWHM}^{26}$ of the hybrid sensor are about half of that for a plain Au film sensor. This is expected since the nanodots will induce some level of scattering loss, while a plain Au film will not.

Table 1. A comparison of the sensitivity of the cylindrical shaped hybrid sensor to that of a plain Au film for a bulk change in the background refractive index.

\begin{tabular}{|l|l|l|l|l|l|l|l|}
\hline & $\begin{array}{l}\text { Resonance } \\
\lambda, \mathbf{n}_{\mathbf{b}}=\mathbf{1 . 3 3 3}\end{array}$ & $\begin{array}{l}\text { Resonance } \\
\lambda, \mathbf{n}_{\mathbf{b}}=\mathbf{1 . 3 4}\end{array}$ & $\boldsymbol{\theta}$ & $\Delta \lambda$ & $\Delta \lambda / \Delta \mathbf{n}$ & FWHM & FOM \\
\hline Hybrid Sensor & $1118 \mathrm{~nm}$ & $1226 \mathrm{~nm}$ & $70^{\circ}$ & $108 \mathrm{~nm}$ & 15429 & $105 \mathrm{~nm}$ & 147 \\
\hline Au Film Sensor & $1118 \mathrm{~nm}$ & $1318 \mathrm{~nm}$ & $68.3^{\circ}$ & $200 \mathrm{~nm}$ & 28571 & $110 \mathrm{~nm}$ & 260 \\
\hline Hybrid Sensor & $852 \mathrm{~nm}$ & $886 \mathrm{~nm}$ & $75^{\circ}$ & $34 \mathrm{~nm}$ & 4857 & $63 \mathrm{~nm}$ & 77 \\
\hline Au Film Sensor & $852 \mathrm{~nm}$ & $917 \mathrm{~nm}$ & $70.7^{\circ}$ & $65 \mathrm{~nm}$ & 9286 & $55 \mathrm{~nm}$ & 168 \\
\hline
\end{tabular}

Having established that the proposed hybrid sensor retains to quite a significant level the total integrated sensitivity, we explore now its surface sensitivity and compare it to the case of the plain Au film. For this we simulate the case where a $3 \mathrm{~nm}$ thick layer of material with refractive index $n_{l}=1.34$ has been added to the sensor surface with $n_{b}=1.333$. Because of drastic increases in computation time, we simulate for the case of the hybrid sensor a $3 \mathrm{~nm}$ thick layer covering just the cylinder, rather than the whole sensor surface. For the Au film the whole surface is covered with the $3 \mathrm{~nm}$ layer. The results, summarized in table 2, show the superiority of the hybrid sensor over the plain Au film. By just adding this $3 \mathrm{~nm}$ layer on top of the cylinders, we are able to induce a $\Delta \lambda / \Delta \mathrm{n}$ shift of $2286 \mathrm{~nm} / \mathrm{RIU}$ and $1143 \mathrm{~nm} / \mathrm{RIU}$ for incidence angles of $70^{\circ}$ and $75^{\circ}$ respectively, while for the plain Au film we obtain values of just $143 \mathrm{~nm} / \mathrm{RIU}$ and $114 \mathrm{~nm} / \mathrm{RIU}$. 
Table 2. A comparison of the surface sensitivity of the cylindrical shaped hybrid sensor to that of a plain Au film for a refractive index change in just a 3 nm layer above the sensor surface.

\begin{tabular}{|l|l|l|l|l|l|l|l|}
\hline & $\begin{array}{c}\text { Resonance } \boldsymbol{\lambda}, \\
\mathbf{n}_{\mathbf{l}}=\mathbf{n}_{\mathbf{b}}=\mathbf{1 . 3 3 3}\end{array}$ & $\begin{array}{c}\text { Resonance } \\
\lambda, \mathbf{n}_{\mathbf{l}}=\mathbf{1 . 3 4}\end{array}$ & $\boldsymbol{\theta}$ & \multicolumn{1}{|c|}{$\Delta \boldsymbol{\Delta}$} & $\Delta \lambda / \Delta \mathbf{n}$ & FWHM & FOM \\
\hline Hybrid Sensor & $1118 \mathrm{~nm}$ & $1134 \mathrm{~nm}$ & $70^{\circ}$ & $16 \mathrm{~nm}$ & 2286 & $105 \mathrm{~nm}$ & 22 \\
\hline Au Film Sensor & $1118 \mathrm{~nm}$ & $1119 \mathrm{~nm}$ & $68.3^{\circ}$ & $1 \mathrm{~nm}$ & 143 & $110 \mathrm{~nm}$ & 1.3 \\
\hline Hybrid Sensor & $852 \mathrm{~nm}$ & $860 \mathrm{~nm}$ & $75^{\circ}$ & $8 \mathrm{~nm}$ & 1143 & $63 \mathrm{~nm}$ & 18 \\
\hline Au Film Sensor & $852 \mathrm{~nm}$ & $852.8 \mathrm{~nm}$ & $70.7^{\circ}$ & $0.8 \mathrm{~nm}$ & 114 & $55 \mathrm{~nm}$ & 2.7 \\
\hline
\end{tabular}

\subsection{Realistic Geometry}

For reasons given in section 4, it is more realistic to model the nanodots as half-domes shown in Fig. 2, rather than as perfect cylinders shown in fig. 1. Exploring both the bulk and surface sensitivity of such a structure in the exact same manner as that of the sensor structure in section 3.1, we arrive at the results shown in table 3 and table 4 . As can be seen, these results suggest that the half-dome geometry allows for essentially the same level of bulk sensitivity, but for a much improved level of surface sensitivity over that of an ideal cylindrical geometry. In the best case, at a resonance of $\lambda=788 \mathrm{~nm}$, the hybrid sensor is able to retain $56 \%$ of the total integrated sensitivity of a plain Au film sensor by achieving a $\mathrm{FOM}=41.5$ with just a $3 \mathrm{~nm}$ thick sensing region on the nanodot surface, while the plain Au film displays a bulk $\mathrm{FOM}=74$. The reason for this can be seen in fig. 6 where we plot the electric field profile for a cross section in the $\mathrm{x}-\mathrm{Z}$ plane cutting directly through the center of the half-dome. In comparison to the case of a cylinder, Fig. 5, the field localization falls mostly along top of the half-dome, rather than at the corners and in the gap between the nanodot and film and is also of a much stronger magnitude.

Table 3. A comparison of the sensitivity of the half-dome shaped hybrid sensor to that of a plain Au film for a bulk change in the background refractive index.

\begin{tabular}{|l|l|l|l|l|l|l|l|}
\hline & $\begin{array}{l}\text { Resonance } \\
\lambda, \mathbf{n}_{\mathbf{b}}=\mathbf{1 . 3 3 3}\end{array}$ & $\begin{array}{l}\text { Resonance } \\
\lambda, \mathbf{n}_{\mathbf{b}}=\mathbf{1 . 3 4}\end{array}$ & $\boldsymbol{\theta}$ & $\Delta \lambda$ & $\Delta \lambda / \Delta \mathbf{n}$ & FWHM & FOM \\
\hline Hybrid Sensor & $1028 \mathrm{~nm}$ & $1124 \mathrm{~nm}$ & $70^{\circ}$ & $96 \mathrm{~nm}$ & 13714 & $105 \mathrm{~nm}$ & 131 \\
\hline Au Film Sensor & $1028 \mathrm{~nm}$ & $1163 \mathrm{~nm}$ & $68.87^{\circ}$ & $135 \mathrm{~nm}$ & 19286 & $66 \mathrm{~nm}$ & 292 \\
\hline Hybrid Sensor & $788 \mathrm{~nm}$ & $820 \mathrm{~nm}$ & $75^{\circ}$ & $32 \mathrm{~nm}$ & 4571 & $62 \mathrm{~nm}$ & 74 \\
\hline Au Film Sensor & $788 \mathrm{~nm}$ & $833 \mathrm{~nm}$ & $71.96^{\circ}$ & $45 \mathrm{~nm}$ & 6429 & $54 \mathrm{~nm}$ & 119 \\
\hline
\end{tabular}

Table 4. A comparison of the surface sensitivity of the half-dome shaped hybrid sensor to that of a plain Au film for a refractive index change in just a 3 nm layer above the sensor surface.

\begin{tabular}{|l|l|l|l|l|l|l|l|}
\hline & $\begin{array}{c}\text { Resonance } \boldsymbol{\lambda}, \\
\mathbf{n}_{\mathbf{l}}=\mathbf{n}_{\mathbf{b}}=\mathbf{1 . 3 3 3}\end{array}$ & $\begin{array}{c}\text { Resonance } \\
\boldsymbol{\lambda}, \mathbf{n}_{\mathbf{l}}=\mathbf{1 . 3 4}\end{array}$ & $\boldsymbol{\theta}$ & $\Delta \lambda$ & $\Delta \lambda / \Delta \mathbf{n}$ & FWHM & FOM \\
\hline Hybrid Sensor & $1028 \mathrm{~nm}$ & $1050 \mathrm{~nm}$ & $70^{\circ}$ & $22 \mathrm{~nm}$ & 3143 & $105 \mathrm{~nm}$ & 30 \\
\hline Au Film Sensor & $1028 \mathrm{~nm}$ & $1028.3 \mathrm{~nm}$ & $68.87^{\circ}$ & $0.3 \mathrm{~nm}$ & 42.9 & $68 \mathrm{~nm}$ & 0.63 \\
\hline Hybrid Sensor & $788 \mathrm{~nm}$ & $806 \mathrm{~nm}$ & $75^{\circ}$ & $18 \mathrm{~nm}$ & 2571 & $62 \mathrm{~nm}$ & 41.5 \\
\hline Au Film Sensor & $788 \mathrm{~nm}$ & $788.9 \mathrm{~nm}$ & $71.96^{\circ}$ & $0.9 \mathrm{~nm}$ & 129 & $47 \mathrm{~nm}$ & 2.74 \\
\hline
\end{tabular}




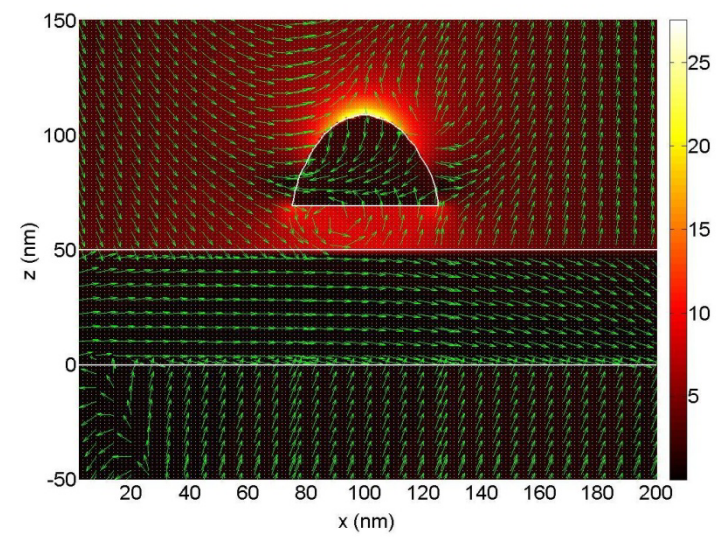

Figure 6. Electric field profile at $70^{\circ}$ incidence for a cross section in the $\mathrm{x}-\mathrm{z}$ plane cutting through the center of the halfdome nanodot. Arrows depict the electric field orientation and the color scale shows the field magnitude.

\section{FABRICATION}

The fabrication of this hybrid biosensor was carried out as follows. First, a $3 \mathrm{~nm}$ adhesion layer of $\mathrm{Cr}$ was evaporated onto a glass substrate, followed directly by a $50 \mathrm{~nm}$ layer of Au. The patterning of the nanodots was then accomplished through a specialized ebeam, evaporation, and lift-off process. This involved a combination of the standard MMAPMMA lift-process, but with an additional step for an added HSQ resist layer. This combination was chosen since MMA and PMMA are positive resists, while HSQ is a negative resist. With an ebeam exposure the written sections of MMAPMMA are etched away, while the written sections HSQ are not. We start with a spin coating of three resist layers in the order HSQ, MMA, PMMA. Following this we perform an ebeam write and development of the MMA-PMMA layers, thereby resulting in open regions in which the Au nanodots can be deposited. Next we evaporate a $2 \mathrm{~nm}$ Ti adhesion layer, followed directly by a 40nm layer of Au and subsequently a lift-off of the MMA-PMMA layers, leaving behind $\mathrm{Au}$ nanodots on the HSQ layer. As a finishing step, the HSQ layer is developed and a final liftoff step is completed resulting in the final structure depicted in section 2. Note, $\mathrm{HSQ}$ was chosen as a spacer layer since it transforms into $\mathrm{SiO}_{2}$ after exposure and development and also because it provides much better adhesion of the Au nanodots than a simple evaporation of $\mathrm{SiO}_{2}$ between the Au layers would.

In order to produce very large scale arrays of these structures (on the order of $2 \mathrm{~mm}^{2}$ ) we use a simple, but effective ebeam write method. Since each nanadot is circular in shape when viewed directly above, a single ebeam pixel can be used to write a single nanodot. With this we are able to pattern a $1 \mathrm{~mm}^{2}$ array of such structures in just three minutes. Subsequent SEM scans of the structure array show very good results in terms of nanodot size, periodicity, and large scale uniformity (Fig. 7). As can been seen from the SEM images, the nanodots end up being half domed in shape, rather than cylindrical. This is due to not only the surface tension of $\mathrm{Au}$, but also to the fact that at these small scales its crystalline nature does not allow for the sharp edges of the perfect cylindrical structure.

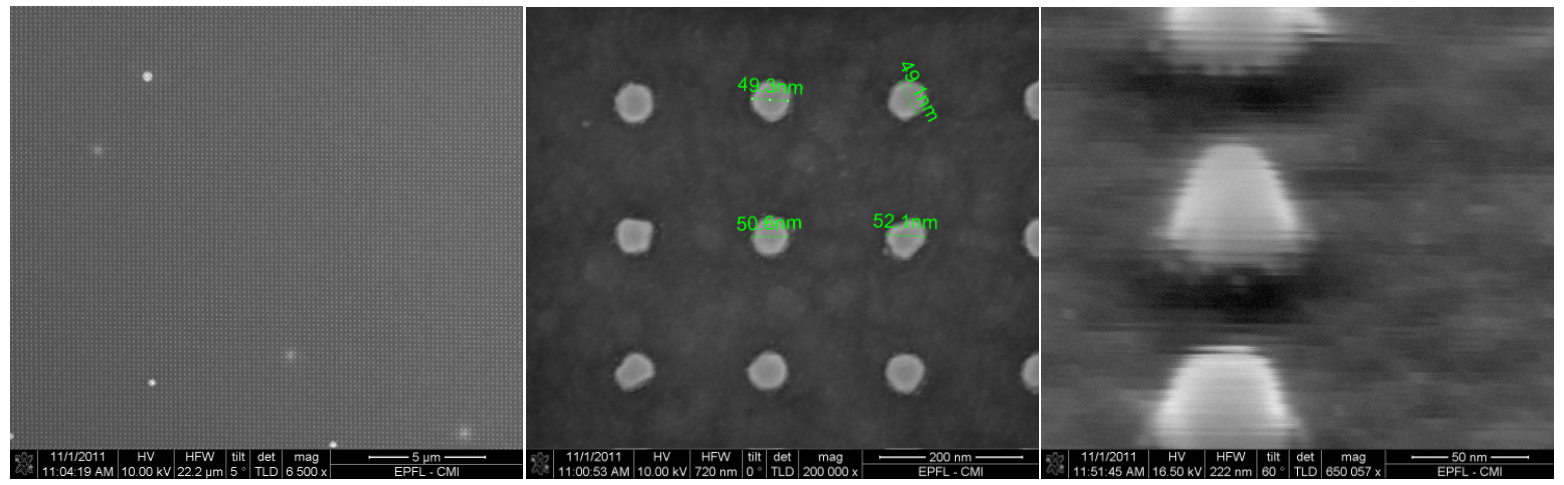

Figure 7. SEM images of the fabricated Au nanodot array, showing the large scale uniformity. The nanodots have the desired $50 \mathrm{~nm}$ diameter and $200 \mathrm{~nm}$ periodicity, but are half-dome rather than cylindrical in shape. 


\section{CONCLUSION}

We have investigated a hybrid plasmonic biosensor composed of a 2D array of Au nanodots coupled to a Au film, which captures both the advantages of SPR and LSPR sensing. Optical excitation via the Kretschmann configuration and a 20nm spacing between the Au nanodots and Au film allows for the nanodots to localize the optical field directly at the sensor surface, without inducing much more than a redshift in the SPP dispersion. With this the sensor is able to retain to a notable degree the total integrated sensitivity of an ideal SPR sensor, while displaying the surface sensitivity of an LSPR sensor. In particular, numerical simulations show that the half-dome shaped nanodots obtained via fabrication achieve up to $56 \%$ of the total integrated sensitivity of a plain Au film sensor with just a $3 \mathrm{~nm}$ sensing layer on the nanodot surface.

\section{REFERENCES}

[1] S. Zhu, T. Y. Liow, G. Q. Lo et al., "Fully complementary metal-oxide-semiconductor compatible nanoplasmonic slot waveguides for silicon electronic photonic integrated circuits," Applied Physics Letters, 98(2), 021107-3 (2011).

[2] S. Sederberg, "Monolithic integration of plasmonic waveguides into a complimentary metal-oxidesemiconductor- and photonic-compatible platform," Applied Physics Letters, 96(12), 3 (2010).

[3] Q. A. Li, Y. Song, G. Zhou et al., "Asymmetric plasmonic-dielectric coupler with short coupling length, high extinction ratio, and low insertion loss," Optics Letters, 35(19), 3153-3155 (2010).

[4] N. Large, M. Abb, J. Aizpurua et al., "Photoconductively Loaded Plasmonic Nanoantenna as Building Block for Ultracompact Optical Switches," Nano Letters, 10(5), 1741-1746 (2010).

[5] H. S. Chu, "Optical performance of single-mode hybrid dielectric-loaded plasmonic waveguide-based components," APPLIED PHYSICS LETTERS, 96(22), 3 (2010).

[6] J. S. Huang, T. Feichtner, P. Biagioni et al., "Impedance Matching and Emission Properties of Nanoantennas in an Optical Nanocircuit," Nano Letters, 9(5), 1897-1902 (2009).

[7] Y. Bian, Z. Zheng, X. Zhao et al., "Symmetric hybrid surface plasmon polariton waveguides for 3D photonic integration," Opt. Express, 17(23), 21320-21325 (2009).

[8] A. a. N. Alu, Engheta, "Optical nanoswitch: an engineered plasmonic nanoparticle with extreme parameters and giant anisotropy," New Journal of Physics, 11(1), 013026 (2009).

[9] A. L. Pyayt, B. Wiley, Y. N. Xia et al., "Integration of photonic and silver nanowire plasmonic waveguides," Nature Nanotechnology, 3(11), 660-665 (2008).

[10] T. Holmgaard, Z. Chen, S. I. Bozhevolnyi et al., "Bend- and splitting loss of dielectric-loaded surface plasmonpolariton waveguides," Opt. Express, 16(18), 13585-13592 (2008).

[11] T. Holmgaard, S. I. Bozhevolnyi, L. Markey et al., "Dielectric-loaded surface plasmon-polariton waveguides at telecommunication wavelengths: Excitation and characterization," Appl. Phys. Lett., 92, 011124 (2008).

[12] H. Fischer, and O. J. F. Martin, "Engineering the optical response of plasmonic nanoantennas," Optics Express, 16(12), 9144-9154 (2008).

[13] S. I. Bozhevolnyi, V. S. Volkov, E. Devaux et al., "Channel plasmon subwavelength waveguide components including interferometers and ring resonators," Nature, 440(7083), 508-511 (2006).

[14] M. Nezhad, K. Tetz, and Y. Fainman, "Gain assisted propagation of surface plasmon polaritons on planar metallic waveguides," Opt. Express, 12(17), 4072-4079 (2004).

[15] D. K. Gramotnev, and S. I. Bozhevolnyi, "Plasmonics beyond the diffraction limit," Nature Photonics, 4(2), 8391.

[16] J. Bhattacharya, "A photonic-plasmonic structure for enhancing light absorption in thin film solar cells," Applied Physics Letters, 99(13), 131114 (2011).

[17] R. A. Pala, E. S. Barnard, J. S. White et al., "Plasmonic solar cells with broadband absorption enhancements," Lasers and Electro-Optics/Quantum Electronics and Laser Science Conference: 2010 Laser Science to Photonic Applications, CLEO/QELS 2010.

[18] M. C., "Enhancement of optical absorption in thin-film organic solar cells through the excitation of plasmonic modes in metallic gratings," APPLIED PHYSICS LETTERS, 96(13), 3 (2010).

[19] A. J. Morfa, K. L. Rowlen, T. H. I. Reilly et al., "Plasmon-enhanced solar energy conversion in organic bulk heterojunction photovoltaics," Appl. Phys. Lett., 92, 013504 (2008). 
[20] K. R. Catchpole, and A. Polman, "Plasmonic solar cells," Opt. Express, 16(26), 21793-21800 (2008).

[21] Y. A. Akimov, and W. S. Koh, "Design of Plasmonic Nanoparticles for Efficient Subwavelength Light Trapping in Thin-Film Solar Cells," Plasmonics, 6(1), 155-161.

[22] A. M. Gobin, M. H. Lee, N. J. Halas et al., "Near-Infrared Resonant Nanoshells for Combined Optical Imaging and Photothermal Cancer Therapy," Nano Lett., 7(7), 1929-1934 (2007).

[23] J. Chen, D. Wang, J. Xi et al., "Immuno Gold Nanocages with Tailored Optical Properties for Targeted Photothermal Destruction of Cancer Cells," Nano Lett., 7(5), 1318-1322 (2007).

[24] D. P. O'Neal, L. R. Hirsch, N. J. Halas et al., "Photo-thermal tumor ablation in mice using near infraredabsorbing nanoparticles," Cancer Letters, 209(2), 171-176 (2004).

[25] L. R. Hirsch, R. J. Stafford, J. A. Bankson et al., "Nanoshell-mediated near-infrared thermal therapy of tumors under magnetic resonance guidance," Proceedings of the National Academy of Sciences of the United States of America, 100(23), 13549-13554 (2003).

[26] J. Homola, [Surface plasmon resonance based sensors.] Springer, (2006).

[27] N. Verellen, P. Van Dorpe, C. J. Huang et al., "Plasmon Line Shaping Using Nanocrosses for High Sensitivity Localized Surface Plasmon Resonance Sensing," Nano Letters, 11(2), 391-397 (2011).

[28] W. P. Hall, S. N. Ngatia, and R. P. Van Duyne, "LSPR Biosensor Signal Enhancement Using Nanoparticle-Antibody Conjugates," The Journal of Physical Chemistry C, 115(5), 1410-1414 (2011).

[29] K. Kim, D. J. Kim, S. Moon et al., "Localized surface plasmon resonance detection of layered biointeractions on metallic subwavelength nanogratings," Nanotechnology, 20(31), 6 (2009).

[30] F. Hao, Y. Sonnefraud, P. V. Dorpe et al., "Symmetry Breaking in Plasmonic Nanocavities: Subradiant LSPR Sensing and a Tunable Fano Resonance," Nano Lett., 8(11), 3983-3988 (2008).

[31] G. H. Chan, J. Zhao, G. C. Schatz et al., [Localized Surface Plasmon Resonance Spectroscopy of Triangular Aluminum Nanoparticles], (2008).

[32] J. N. Anker, W. P. Hall, O. Lyandres et al., "Biosensing with plasmonic nanosensors," Nature Materials, 7(6), 442-453 (2008).

[33] A. Dahlin, M. Zach, T. Rindzevicius et al., "Localized surface plasmon resonance sensing of lipid-membranemediated biorecognition events," Journal of the American Chemical Society, 127(14), 5043-5048 (2005).

[34] A. J. Haes, and R. P. Van Duyne, "A unified view of propagating and localized surface plasmon resonance biosensors," Analytical and Bioanalytical Chemistry, 379(7), 920-930 (2004).

[35] M. Svedendahl, S. Chen, A. Dmitriev et al., "Refractometric Sensing Using Propagating versus Localized Surface Plasmons: A Direct Comparison," Nano Letters, 9(12), 4428-4433 (2009).

[36] P. B. Johnson, and R. W. Christy, "Optical Constants of the Noble Metals," Phys. Rev. B, 6(12), 4370-4379 (1972).

[37] G. Lévêque, and O. J. F. Martin, "Optical interactions in a plasmonic particle coupled to a metallic film," Optics Express, 14(21), 9971-9981 (2006).

[38] F. Le, N. Z. Lwin, N. J. Halas et al., "Plasmonic interactions between a metallic nanoshell and a thin metallic film," Physical Review B, 76(16), 165410 (2007).

[39] P. Nordlander, and E. Prodan, "Plasmon Hybridization in Nanoparticles near Metallic Surfaces," Nano Letters, 4(11), 2209-2213 (2004).

[40] S. M. Jang, D. Kim, S. H. Choi et al., "Enhancement of localized surface plasmon resonance detection by incorporating metal-dielectric double-layered subwavelength gratings," Applied Optics, 50(18), 2846-2854 (2011).

[41] B. Gallinet, A. M. Kern, and O. J. F. Martin, "Accurate and versatile modeling of electromagnetic scattering on periodic nanostructures with a surface integral approach," J. Opt. Soc. Am. A, 27(10), 2261-2271 (2010).

[42] Y. Chu, and K. B. Crozier, "Experimental study of the interaction between localized and propagating surface plasmons," Optics Letters, 34(3), 244-246 (2009).

[43] A. Ghoshal, I. Divliansky, and P. G. Kik, "Experimental observation of mode-selective anticrossing in surfaceplasmon-coupled metal nanoparticle arrays," Applied Physics Letters, 94(17), 171108-3 (2009).

[44] W. R. Holland, and D. G. Hall, "Surface-plasmon dispersion relation: Shifts induced by the interaction with localized plasma resonances," Physical Review B, 27(Copyright (C) 2009 The American Physical Society), 7765 (1983).

[45] N. Papanikolaou, "Optical properties of metallic nanoparticle arrays on a thin metallic film," Physical Review B, 75(23), 235426 (2007). 